International Communications in Heat and Mass Transfer (ELSEVIER, USA)

Cite score 8.7, Impact Factor, 3.971

Editor-in-Chief: Prof. Dr. William Worek, Texas A\&M University Kingsville Eagle Ford Center for Research and Outreach, 700 University Blvd. - MSC 188, Kingsville, Texas, 78363, United States.

Accepted January $25^{\text {th }} 2021$

\title{
MATHEMATICAL MODELLING OF UNSTEADY SOLUTE (OXYGEN) DISPERSION IN TWO-FLUID (MICROPOLAR-NEWTONIAN) BLOOD FLOW WITH BULK REACTION
}

\author{
Ashis Kumar Roy ${ }^{\mathbf{a} *}$ and O. Anwar Bég \\ ${ }^{a}$ Lecturer, Non-Newtonian Hydrodynamics Group, Applied Mathematics Division, Department of Science \\ \& Humanities, Tripura Institute of Technology, Agartala, Tripura-799009, India \\ ${ }^{b}$ Professor, Multi-Physical Engineering Sciences Group, Mechanical Engineering Department, School of \\ Science, Engineering and Environment (SEE), University of Salford, Manchester-M54WT, UK
}

*Corresponding Author: Email: rk.ashis10@gmail.com

ABSTRACT - A mathematical model is developed for axisymmetric, incompressible, and fully developed hemodynamic transport of a reactive diffusing species, e. g. oxygen, in a rigid, impermeable artery under constant axial pressure gradient which undergoes a first-order chemical reaction with streaming blood. A two-fluid model is deployed where the core region is simulated as an Eringen micropolar fluid, and the plasma layer engulfing the core, i.e., near the boundary, is analyzed as a Newtonian viscous fluid. At the interface of the core and plasma region, the velocity and shear stress are equal, and micro-rotation is constant. Closed-form solutions are presented for the velocity and micro-rotation profiles, and a Gill decomposition method is deployed for the concentration field. Expressions are derived for the dispersion coefficient, mean and transverse concentration functions. Transverse concentration is observed to be enhanced with increasing micropolar coupling number $(N)$ and reaction rate $(\beta)$; however, it is reduced with greater micropolar material parameter $(s)$ and viscosity ratio $(\lambda)$. Additionally, graphs are presented for the evolution in dispersion coefficient, and the rate of dispersion coefficient with micropolar parameters is examined. Finally, both axial and transverse mean concentration distributions for all key parameters are investigated. Transverse concentration is observed to be enhanced with increasing micropolar coupling number and reaction rate; however, it is reduced with greater micropolar material parameter and viscosity ratio. Axial mean concentration peaks are reduced in magnitude and displaced further along the arterial geometry with greater micropolar material parameter values, whereas the opposite effect is induced with greater micropolar coupling number. A slight increase in axial mean concentration peak value is computed with increasing reaction parameter. The dispersion coefficient is reduced with increasing micropolar material parameter but grows with a greater viscosity ratio. The study is relevant to hemorheology, diseased arteries and coagulating hemodynamics and may help physiologists and clinicians in furnishing a more refined understanding of diffusion processes in cardiovascular hydrodynamics.

KEYWORDS - Hemo-rheology; Micropolar model; Newtonian model; Unsteady hydrodynamic dispersion; Bulk chemical reaction; Coagulation; Convective diffusion. 


\begin{tabular}{|c|c|c|c|}
\hline \multicolumn{4}{|c|}{ NOMENCLATURE } \\
\hline$T_{i j}$ & Cauchy stress tensor & $\mu_{1}^{\prime}$ & $\begin{array}{l}\text { dynamic viscosity coefficient } \\
\text { of the micropolar fluid. }\end{array}$ \\
\hline$\Gamma_{i j}$ & couple stress tensor & $\mu_{2}^{\prime}$ & dynamic viscosity of the \\
\hline & & & Newtonian fluid. \\
\hline$\delta_{i j}$ & Kronecker delta & $U$ & reference velocity \\
\hline$\varepsilon_{i j k}$ & alternating tensor & $N$ & micropolar coupling number \\
\hline$p$ & isotropic pressure & $\eta$ & core radius to total radius ratio \\
\hline$v_{i}$ & components of velocity vector & $\lambda$ & viscosity ratio. \\
\hline$\omega_{i}$ & components of vorticity vector & $s^{2}$ & micropolar material parameter \\
\hline$w_{i}$ & $\begin{array}{l}\text { components of micro-rotation } \\
\text { vector }\end{array}$ & $\beta^{\prime}$ & first-order chemical reaction \\
\hline$\rho$ & fluid density & $\mathrm{Pe}$ & Péclet number \\
\hline$\mu, \kappa$ & coefficients of viscosity & $K_{1}$ & advection coefficient \\
\hline $\begin{array}{l}\alpha, \beta \\
\text { and } \gamma\end{array}$ & coefficients of gyroviscosity & $K_{2}$ & dispersion coefficient \\
\hline$C^{\prime}$ & species concentration & $J_{0}$ & Bessel function of order zero \\
\hline$M$ & mass of the species & $\bar{C}$ & mean concentration \\
\hline$\delta(\cdot)$ & direct delta function & $R_{K_{2}, \beta}$ & $\begin{array}{l}\text { decrement of dispersion } \\
\text { coefficient due to reaction rate } \\
\text { constant }\end{array}$ \\
\hline
\end{tabular}




\begin{tabular}{|llll|}
\hline$R_{1}^{\prime}$ core radius & $R_{K_{2}, N}$ & decrement of dispersion \\
& & coefficient due to micropolar \\
& & coupling number \\
$R^{\prime} \quad$ radius of the vessel & $R_{K_{2}, \lambda}$ & rate of increment of dispersion \\
& coefficient due to viscosity \\
& ratio \\
&
\end{tabular}

\section{INTRODUCTION}

It has been firmly established in medical sciences for many decades that blood comprises a sophisticated composite suspension of proteins, lipoproteins, ions, white, and red blood cells suspended in plasma (water). Erythrocytes alone constitute $40 \%$ of blood by volume, and owing to the small semi-solid and toroidal geometry of these cells, they result in a non-trivial elevation in blood viscosity of blood - this contributes to the fact that normal human blood is approximately four times more viscous than water[1]. Furthermore, this viscosity varies in the human circulation system and assumes strongly rheological properties in the microcirculatory system $[2,3]$. This has been predicted theoretically and confirmed experimentally [4]. Moreover, strongly non-Newtonian characteristics are observed in small branches and capillary sections of the body, wherein cell-free skimming leads to a decrease in viscosity. These investigations have broadened the science of hemodynamics to create a new field in its own right, namely hemo-rheology, which may be regarded as the study of how the blood, the blood cells, and the vessels can function and interact as parts of the living organism. More specifically, hemorheology or hemodynamic biorheology is the science of deformation and flow of blood and its formed elements. This field includes investigations of both macroscopic blood properties using rheumatic experiments as well as microscopic properties in vitro and in vivo. 
Hemorheology features in numerous human ailments including diabetes mellitus [5], aneurysmal subarachnoid hemorrhage [6], Alzheimer's disease (in which erythrocyte aggregation reduced oxygen transport is observed) [7], thoracic aortic aneurysms and blood clots [8] and coronary perfusion in cardiac syndrome [9]. Many excellent reviews of hemorheology have appeared in recent years, describing in great detail clinical observations of abnormalities. These include Baskurt and Meiselman [10] and Stoltz et al. [11]. Blood flow in the microvasculature is essentially determined by viscous forces (i.e., blood viscosity), as elaborated by Skalak et al. [12]. At low shear rates, even in large arteries, blood exhibits non-Newtonian characteristics. The presence of multiple suspensions (proteins, fats, ions, red and white blood cells, etc.) generates markedly rheological properties in blood. To more precisely simulate shear stress-strain characteristics of actual blood, therefore rheological models are required, and these are invariably adopted from polymer physics and chemical engineering for which they were originally developed [13]. The simpler and more popular rheological models for hemodynamics, e.g., shear-thinning, power-law, viscoplastic (yield stress), e.g., Casson, Quemada models etc. have been summarized by Robertson et al. [14] and shown to predict reasonably well the shear flow behavior of blood in leukemia, haemolytic anemia, and thalassemia conditions. However, many more complex models are available, which can simulate viscoelasticity, retardation, relaxation, memory, and other rheological effects in blood. These include the Oldroyd-B elastic, viscous model, Maxwell upper convected (UCM) viscoelastic models, FENE-P (finitely extensible nonlinear elasticPeterlin) model, Williamson fluids, Walters-B short memory fluid model etc. However, these models cannot simulate the microstructural characteristics of streaming blood. Several models have, however, been developed which analyze the microstructural feature of blood and other complex liquids. These include the polar (Stokes couple stress) model 
[15,16] and the more comprehensive Eringen micromorphic model [17]. The most generalized form of the micromorphic model includes gyration (microrotation), i.e., the spin of microelements, couple stresses, and also axial contractions/extensions. It is, however, a very complex formulation and has been employed in only several studies of blood flow, notably Narashimhan [18] and Philip and Chandra [19]. A much simpler but still robust subset of the microfluid or micromorphic fluids is the micropolar model, also introduced by Eringen [20]. These fluids feature a non-symmetric tensor, intrinsic motion, and local microstructures. Early work in blood flows using the micropolar model [21] has shown that They can successfully explain key hemodynamic anomalies, e.g., the Segre-Silberberg effect, Fahraeus-Lindquist effect, and blunting of velocity profiles, whereas alternate non-Newtonian models, at best, can only reproduce one or two of these anomalies. Many studies have, therefore, explored theoretical and numerical micropolar blood simulation in the past four decades. Chataurani and Mahajan [22] investigated the micropolar Poiseuille blood flow analytically with non-zero couple stresses at the vessel boundary. They derived expressions for axial and rotational (micro-rotation) velocity components and also apparent viscosity and validated with experimental values (for blood flow). Akay and Kaye [23] studied the time-dependent stratified two-phase micropolar blood flow in small diameter capillaries using two different spin boundary conditions. Chaturani and Palanisamy [24] analyzed the micropolar blood flow in a stenotic arterial geometry using finite Hankel and Laplace transforms. They observed that micropolarity accelerates the flow in certain locations (compared with Newtonian fluid) and furthermore reproduced the apparent viscosity variation accurately with tube radius, i.e., inverse Fahraeus-Lindqvist and Fahraeus-Lindqvist effects. Mekheimer and Elmaboud [25] simulated the micropolar blood clot dynamics in annular peristaltic flow in small diameter vessels, also computing pressure rise and friction force on the inner and the outer 
tubes with different clot heights. Bég et al. [26] used variational finite element and finite difference codes to compute the biomagnetic micropolar convective blood flow in perfused tissue regions, computing wall couple stresses, hemodynamic impedance, and microrotation contours for a range of gyroviscosity and coupling parameters. Sarifuddin et al. [27] employed a Marker and Cell (MAC) method to simulate the transient micropolar blood flow and heat transfer through constricted rigid arteries. They computed wall shear stress, streamlines, separation-reattachment points, pressure drop, and temperature contours, noting that non-zero micro spin velocity on the arterial wall leads to early flow separation and that excess pressure drop arises across the cosine-form stenoses compared with other irregular geometries. Rashidi et al. [28] used a differential transform method (DTM) to compute the magnetized micropolar blood flow in free convection hemodynamic filtration systems. Further studies deploying the micropolar model in a variety of biological transport problems include Ali et al. [29] (bacterial swimming slime dynamics), Chaube et al. [30] (electro-kinetic biomicrofluidic devices using non-Newtonian fluent media) and Akbar et al. [31] (cilia-generated pumping of micropolar physiological fluids in channels). All these studies have identified the exceptional versatility of Eringen's micropolar model in modelling more precisely hemodynamic flows and also a range of other biophysical fluid dynamics phenomena.

In hemodynamics, biochemical reactions arise frequently. These are associated with diffusing constituents, e.g., oxygen reacting, and also other phenomena such as coagulation, which is a rigorously regulated biochemical network of reactions (featuring includes zymogens, enzymes, co-factors, inhibitors etc.) which leads to the generation of thrombin at the site of an injury and simultaneously eradicate it in the peripheral area [32]. Chemo-hemodynamics is, therefore, of considerable interest in more detailed simulations of real blood flows and has received some attention in recent years. 
Interesting clinical studies include Mann et al. [33] (on surfacial reactions in blood), Mann et al. [34] (on coagulation chemical reactions on the subendothelium and endothelial cells), Mann [35](on physico-chemical coagulation in thromboses), Shen et al. [36] (on diffusion-reaction in blood clotting), Zhao et al. [37] (chemo-hemodynamic regulation using nitric oxide synthase (NOS) inhibitor in arterialized venous flaps). A number of mathematical models have also been developed for studying reactive hydrodynamics of blood flows, and these also feature dispersion effects (usually modelled with modifications of the classical Taylor theory). $\mathrm{Ng}$ [38] developed an asymptotic method of averaging to study reactive blood flow with an extended Aris dispersion model. He computed the impact of the second-order effects of the reaction on the advection velocity and the reaction rate itself. Murthy and Feyen [39] have examined theoretically the dual influence of variable permeability and homogeneous and heterogeneous chemical reactions on the dispersion of a solute in hydromagnetic blood flow through a conduit containing sparsely and randomly packed porous media. They found that for both diffusions with a first-order homogeneous reaction or homogeneous and heterogeneous chemical reactions, the variation of permeability increases the effective dispersion coefficient, whereas it reduces it with a stronger magnetic field. These studies were confined to Newtonian reactive blood flows. Roy et al. [40,41] however recently investigated solute dispersion in non-Newtonian Casson fluid flow using the Aris-Barton approach. Debnath et al. [42] very recently investigated the dispersion of a reactive solute in steady and furthermore studied oscillatory flows [43,44] of a two-fluid, three-layer Casson-Newtonian continuum with the Aris-Barton approach. They considered blood in the central region to be a Casson viscoplastic fluid (a core of red blood cells in suspension) and a peripheral layer of plasma modelled as a Newtonian fluid. They employed perturbation methods to derive the velocity profiles and a finite difference numerical 
technique for effective dispersion coefficient by solving the moment equations. Furthermore, they used a Hermite polynomial representation of central moments to compute the axial distribution of mean concentration. Micropolar diffusion and reactive flows have also received some attention. Siddheshwar and Markande [45] studied using asymptotic long time analysis, the transient convective diffusion of a species in micropolar blood flow. They derived a time-dependent expression for the exchange coefficient, which was shown to be independent of the velocity distribution of the flow but dependent on the initial solute distribution. Vidyanidhi and Murty [46] analyzed the dispersion of a solute in a micropolar fluid flow in a tube with an irreversible first-order chemical reaction, observing that the Taylor dispersion coefficient decreases with an increase in coupling micropolar parameter whereas it increases with micropolar material parameter values. Further studies include Soundalgekar and Haldavnekar [47] (solute dispersion with a harmonically varying concentration in micropolar channel flow), Siddheshwara and Manjunath [48] (on transient convective diffusion with heterogeneous catalytic chemical reaction in micropolar plane-Poiseuille flow). These investigations all confirmed a significant deviation in transport characteristics computed with the micropolar model relative to the conventional Newtonian model.

In the present study, a novel mathematical model is developed for axisymmetric, incompressible and fully developed hemodynamic convective diffusive transport in a rigid, impermeable artery under constant axial pressure gradient with unsteady reactive hydrodynamic dispersion- as a simulation of reactive oxygen behaviour in rheolgical arterial blood flow. In this study the following methodology is deployed. First a twofluid model is deployed where the core region is simulated as an Eringen micropolar fluid, and the plasma layer is analyzed as a Newtonian viscous fluid. Second, the diffusing species, e.g., oxygen, is studied which undergoes a first-order chemical reaction with 
streaming blood. Third, analytical solutions are developed for the non-dimensional boundary value problem. This involves the derivation of closed-form solutions for the velocity and micro-rotation (angular velocity of micro-elements) profiles, and a Gill decomposition method [49,50] for the concentration field. Fourth, expressions are derived for the dispersion coefficient with reaction and without reaction and also the mean and transverse concentration functions. Fifth, the influence of micropolar coupling number $(N)$, micropolar parameter $(s)$, viscosity ratio (ratio of micropolar viscosity to Newtonian viscosity) (i.e. $\lambda$ ), reaction rate parameter $(\beta)$ on linear (axial) velocity, dispersion coefficient $\left(K_{2}\right)$ rate of dispersion coefficient, axial and transverse mean concentration distributions is visualized graphically. Specifically, the novelty of the present work is therefore the combination of multiple features which have only been addressed individually in previous studies. Herein we amalgamate (i) a two-fluid model with micropolar core region and Newtonian plasma layer, (ii) a first-order chemical reaction model and (iii) the Gill decomposition method for the concentration field. This multi-faceted simulation therefore addresses simultaneously multiple hemo-rheological and physico-chemical characteristics of streaming blood. The study is relevant to diseased hemodynamics and coagulation in blood flow associated with chemical reactions.

\section{MICROPOLAR RHEOLOGICAL MODEL}

In this study, both Newtonian and micropolar fluids are examined to stimulate blood flow. The constitutive equations for micropolar fluids in the tensorial form are:

$$
\begin{gathered}
v_{i, i}=0, \\
\rho v_{j} v_{i, j}=T_{i j, j},
\end{gathered}
$$




$$
\rho j v_{j} w_{i, j}=\Gamma_{i j, j}+\varepsilon_{i j k} T_{j k} \text {, }
$$

Here the linear constitutive laws take the forms $[51,52]$.

$$
\begin{gathered}
T_{i j}=-\rho \delta_{i j}+\frac{1}{2}(2 \mu+\kappa)\left(v_{i, j}+v_{j, i}\right)+\kappa \varepsilon_{i j k}\left(\omega_{k}-w_{k}\right), \\
\Gamma_{i j}=\alpha w_{k, k} \delta_{i j}+\beta w_{i, j}+\gamma w_{j, i},
\end{gathered}
$$

where $T_{i j}$ and $\Gamma_{i j}$ are Cauchy stress tensor and couple stress tensor, respectively. $\delta_{i j}$ is the Kronecker delta, $\varepsilon_{i j k}$ is the alternating tensor, $\mathrm{p}$ is the isotropic pressure, $v_{i}$ are the components of velocity vector, $\omega_{i}$ are the components of vorticity vector $\left(\omega_{i}=(1 / 2) \varepsilon_{i j k} v_{k, j}\right), w_{i}$ are the components of micro-rotation vector $\mathrm{w}$ and $\rho$ and $j$ are the fluid density (blood) and micro-gyration parameter and a comma after a suffix denotes covariant differentiation. Furthermore, the coefficients of viscosity $\mu, \kappa$ and the coefficients of gyro-viscosity $\alpha, \beta$ and $\gamma$ satisfy the following inequalities [52]:

$2 \mu+\kappa \geq 0, \kappa \geq 0,3 \alpha+\beta+\gamma \geq 0, \gamma \geq|\beta|$.

For the flow under consideration, the velocity field is given by $\mathbf{u}=(u, v, 0)$, and the micro-rotation vector is $\mathbf{w}=(0,0, w)$. Owing to the solenoidal nature of the microrotation vector, the coefficient of gyro-viscosity $\alpha$ and $\beta$ do not appear in the governing equations. Moreover, these equations reduce to the classical Navier-Stokes equations (for the Newtonian case) when $N, \varepsilon$ and $j$ vanish.

\section{REACTIVE DISPERSION TWO-FLUID BLOOD FLOW MODEL}

As illustrated in Fig. 1, the regime under consideration studies solute dispersion e. g. oxygen, in fully developed incompressible viscous blood flow through a rigid artery of radius $R^{\prime}$. The model developed amalgamates for the first time, the previous formulations of Chataurani and Mahajan [22] (micropolar hemodynamics), Debnath et al. [42] (micropolar transport in rigid arteries), Siddheshwar and Markande [45] (convective diffusion in micropolar fluids) and Vidyanidhi and Murty [46] (reactive Taylor dispersion in micropolar fluids). 


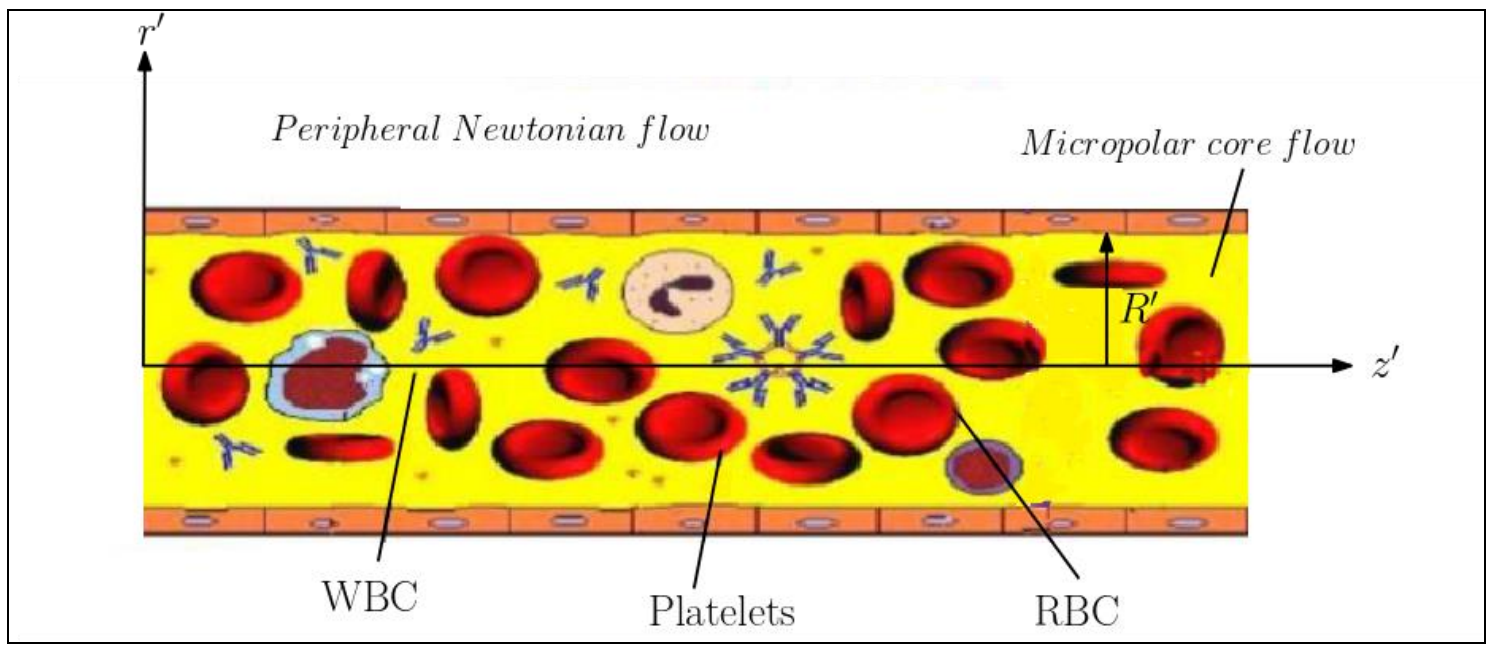

Fig. 1 Two-fluid blood flow in a rigid arterial segment with bulk chemical reaction

The concentration $C^{\prime}\left(z^{\prime}, r^{\prime}, t^{\prime}\right)$ of the species dissolved in the blood is governed by the following partial differential equation:

$$
\frac{\partial C^{\prime}}{\partial t^{\prime}}+u^{\prime}\left(r^{\prime}\right) \frac{\partial C^{\prime}}{\partial z^{\prime}}=D \frac{\partial^{2} C^{\prime}}{\partial z^{\prime 2}}+\frac{D}{r^{\prime}} \frac{\partial}{\partial r^{\prime}}\left(r^{\prime} \frac{\partial C^{\prime}}{\partial r^{\prime}}\right)-\beta^{\prime} C^{\prime} \text { in } 0<r^{\prime}<R^{\prime},
$$

here $D$ denotes constant molecular diffusivity of the species (e. g. oxygen) in blood, $u^{\prime}\left(r^{\prime}\right)$ is blood velocity. Also, the species undergoes a first order chemical reaction with flowing blood at a constant rate $\beta^{\prime}$. The blood vessel walls in the present study is assumed to be impermeable, i.e.,

$$
\cdot \frac{\partial C^{\prime}}{\partial r^{\prime}}=0 \quad \text { at } \quad r^{\prime}=R^{\prime}
$$

Also, due to symmetry:

$$
\frac{\partial C^{\prime}}{\partial r^{\prime}}=0 \quad \text { at } \quad r^{\prime}=0 .
$$

The initial and upstream and downstream conditions are taken as:

$$
\begin{aligned}
& C^{\prime}\left(z^{\prime}, r^{\prime}, 0\right)=\frac{M \delta\left(z^{\prime}\right)}{\pi R_{0}^{\prime 2}}, \\
& C^{\prime}\left( \pm \infty, r^{\prime}, t^{\prime}\right)=0 .
\end{aligned}
$$

Where $M$ is the mass of the species released in the blood flow and $\delta(\cdot)$ is the direct delta function. The blood considered in this study is simulated with a two-fluid model where 
the core region $\left(0<r^{\prime}<R_{1}^{\prime}\right)$ be is modelled by as an Eringen micropolar fluid (containing various suspensions as shown in fig. 1), whereas the plasma layer $\left(R_{1}^{\prime}<r^{\prime}<R^{\prime}\right)$ near the boundary is taken as Newtonian fluid. Constant axial pressure gradient is assumed. We suppose that in both core and plasma layers, the fluid is steady, axisymmetric, incompressible, and fully developed. We denote $\left(0,0, v_{i}^{\prime}\left(r^{\prime}\right)\right), i=1,2$, the velocity vector, where the subscript 1 indicates the velocity in the core region (micropolar zone), whereas subscript 2 represents the velocity in the plasma region (Newtonian zone). The microcirculation velocity of the micropolar fluid in the core region is represented by $\left(0, w^{\prime}\left(r^{\prime}\right), 0\right)$. Thus, the governing equation for fluid flow in the core region:

$$
\begin{aligned}
& -\frac{\partial p^{\prime}}{\partial z^{\prime}}+\left(\mu_{1}^{\prime}+\kappa^{\prime}\right) \frac{1}{r^{\prime}} \frac{d}{d r^{\prime}}\left(r^{\prime} \frac{d v_{1}^{\prime}}{d r^{\prime}}\right)+\frac{\kappa^{\prime}}{r^{\prime}} \frac{d}{d r^{\prime}}\left(r^{\prime} \omega^{\prime}\right)=0,0<r^{\prime}<R_{1}^{\prime} \\
& \gamma^{\prime} \frac{d}{d r^{\prime}}\left(\frac{1}{r^{\prime}} \frac{d}{d r^{\prime}}\left(r^{\prime} w^{\prime}\right)\right)-\kappa^{\prime}\left(\frac{d v_{1}^{\prime}}{d r^{\prime}}+2 w^{\prime}\right)=0,0<r^{\prime}<R_{1}^{\prime}
\end{aligned}
$$

Where $\kappa^{\prime}$ and $\gamma^{\prime}$ are the micropolar fluid viscosity and gyroviscosity coefficients and $\mu_{1}^{\prime}$ is the dynamic viscosity coefficient of the micropolar fluid.

The governing equation for the fluid flow in the plasma region is:

$$
-\frac{\partial p^{\prime}}{\partial z^{\prime}}+\frac{\mu_{2}^{\prime}}{r^{\prime}} \frac{d}{d r^{\prime}}\left(r^{\prime} \frac{d v_{2}^{\prime}}{d r^{\prime}}\right)=0, R_{1}^{\prime}<r^{\prime}<R
$$

The quantity, $\mu_{2}^{\prime}$ in Eq. (8), is the dynamic viscosity of the Newtonian fluid.

\section{NON-DIMENSIONALISATION OF MODEL}

Throughout the remainder of the analysis, the following dimensionless quantities are adopted:

$$
\begin{aligned}
& t=\frac{D t^{\prime}}{R^{\prime 2}}, \quad r=\frac{r^{\prime}}{R^{\prime}}, \quad z^{\prime}=\frac{z}{R^{\prime}}, \quad C=\frac{\pi R^{\prime 3} C^{\prime}}{M}, \quad v_{i}=\frac{v_{i}^{\prime}}{U}(i=1,2), \\
& w=\frac{w^{\prime} R^{\prime}}{U}, \quad N=\frac{\kappa^{\prime}}{\mu_{1}^{\prime}+\kappa^{\prime}}, \quad \eta=\frac{R_{1}^{\prime}}{R^{\prime}}, \quad \lambda=\frac{\mu_{1}^{\prime}}{\mu_{2}^{\prime}}
\end{aligned}
$$


$U=-\left(R^{\prime 2} / \mu_{1}^{\prime}\right) \partial p^{\prime} / \partial z^{\prime}$ is a reference velocity, $N$ indicate the micropolar coupling number, $\eta$ is core radius to total radius ratio and $\lambda$ is the viscosity ratio. Using the scaling as defined in Eq. (15), the initial-boundary value problem (IBVP) given by Eqns. (12-14) emerges as follows:

$$
\begin{gathered}
\frac{1}{r} \frac{d}{d r}\left(r \frac{d v_{1}}{d r}\right)+\frac{N}{r} \frac{d}{d r}(r \omega)+(1-N)=0,0<r<\eta \\
\frac{d}{d r}\left(\frac{1}{r} \frac{d}{d r}(r w)\right)-\frac{s^{2}}{2-N}\left(\frac{d v_{1}}{d r}+2 w\right)=0,0<r<\eta \\
\frac{1}{r} \frac{d}{d r}\left(r \frac{d v_{2}}{d r}\right)+\lambda=0, \eta<r<1
\end{gathered}
$$

Here $s^{2}=\kappa^{\prime} R^{\prime 2}\left(2 \mu_{1}^{\prime}+\kappa^{\prime}\right) / \gamma^{\prime}\left(\mu_{1}^{\prime}+\kappa^{\prime}\right)$ is the micropolar material parameter, $r$ is dimensional radial coordinate. For the present problem the flow boundary conditions are adopted as follows:

(I) At the centre of the artery, the fluid velocity is finite, i.e.,

$$
v_{1}, w<\infty \text { at } r=0
$$

(II) At the interface of the core and plasma region, the velocity and shear stress are the same. Also the micro-rotation (angular velocity) at the interface is constant. Thus,

$$
\begin{gathered}
v_{1}=v_{2} \quad \text { at } \quad r=\eta \\
\frac{d v_{1}}{d r}+N w=\frac{1-N}{\lambda} \frac{d v_{2}}{d r} \quad \text { at } \quad r=\eta \\
\frac{1}{r} \frac{d}{d r}(r w)=0 \quad \text { at } \quad r=\eta
\end{gathered}
$$

(III) No-slip boundary condition at the boundary of the plasma layer (vessel 
wall periphery) leads to:

$$
v_{2}=0 \text { at } r=1
$$

Similarly, the dimensionless form of the convection-diffusion Eq. (7) with associated initial and boundary conditions (8-11) is transformed by virtue of Eq. (15) to:

$$
\begin{aligned}
& \frac{\partial C}{\partial t}+\operatorname{Pe} u(r) \frac{\partial C}{\partial z}=\frac{1}{r} \frac{\partial}{\partial r}\left(r \frac{\partial C}{\partial r}\right)+\frac{\partial^{2} C}{\partial z^{2}}-\beta C, \\
& C(0, r, z)=\delta(z), \quad(0<r<1), \\
& \frac{\partial C}{\partial r}=0 \quad \text { at } \quad r=0, \\
& \frac{\partial C}{\partial r}=0 \quad \text { at } \quad r=1, \\
& \frac{\partial C}{\partial r}=C(t, r, z)=0, \quad \text { at } z \rightarrow \pm \infty .
\end{aligned}
$$

Here $\beta=\beta^{\prime} R^{\prime 2} / D$ is the dimensionless bulk reaction rate and $\operatorname{Pe}\left(=U R^{\prime} / D\right)$ is the effective Péclet number, which quantifies the relative contribution of convection to diffusion.

\section{NEWTONIAN LINEAR \& ERINGEN SPIN MICRO-ROTATION VELOCITY PROFILES}

Now by solving the coupled equation (16) and (18) subject to boundary condition (19) (23) we obtain the solutions for the linear velocity and microrotation in the core region $\left(v_{1}, w\right)$ and linear velocity in the plasma region $\left(v_{2}\right)$ :

$$
v_{1}=\frac{\lambda}{4}\left(1-\eta^{2}\right)+\frac{1-N}{2(2-N)}\left[\eta^{2}-r^{2}+\frac{2 N}{s^{2}}\left(\frac{I_{0}(s r)}{I_{0}(s \eta)}-1\right)\right], \quad 0<r<\eta
$$




$$
\begin{gathered}
w=\frac{1-N}{2(2-N)}\left[r-\frac{2 I_{1}(s r)}{s I_{0}(s \eta)}\right], \quad 0<r<\eta \\
v_{2}=\frac{\lambda}{4}\left(1-r^{2}\right), \quad \eta<r<1
\end{gathered}
$$

If $(\eta, \lambda) \rightarrow(1,1)$ then this two-fluid model is reduced to the single fluid (micropolar) model having following velocity components i.e.

$$
\begin{gathered}
v_{1}=\frac{1-N}{2(2-N)}\left[1-r^{2}+\frac{2 N}{s^{2}}\left(\frac{I_{0}(s r)}{I_{0}(s)}-1\right)\right], \quad 0<r<1 \\
w=\frac{1-N}{2(2-N)}\left[r-\frac{2 I_{1}(s r)}{s I_{0}(s)}\right], \quad 0<r<1
\end{gathered}
$$

Further, if $(\eta, s) \rightarrow(0, \infty), \lambda=1$, and $N=0$, then, Eq. (29) original core flow becomes:

$$
v_{1}=\frac{1}{4}\left(1-r^{2}\right), \quad 0<r<1
$$

This corresponds to classical Newtonian Poiseuille flow

\section{GENERALIZED DISPERSION MODEL}

Following Gill [49], the species concentration field can be decomposed in terms of mean concentration as follows:

$$
C(t, r, z)=\bar{C}+\sum_{i=0}^{\infty} g_{i}(r, t) \frac{\partial^{i} \bar{C}}{\partial z^{i}}
$$

Where $\bar{C}=2 \int_{0}^{1} r C(t, r, z) d r$ indicates the mean concentration of a species. By assuming that the behaviour of mean concentration $\bar{C}$ is diffusive in nature right from the beginning, then accordingly, the mean concentration can be expressed as follows: 


$$
\frac{\partial \bar{C}}{\partial t}=\sum_{i=1}^{\infty} K_{i}(t) \frac{\partial^{i} \bar{C}}{\partial z^{i}},
$$

The terms $K_{1}$ and $K_{2}$ in Eq. (36) are termed as the advection and dispersion coefficient, respectively. Using Eq. (35) in Eq. (36) and Eq. (24) and after some algebraic manipulation, we obtain the following system of partial differential equations:

$$
\begin{gathered}
\frac{\partial g_{1}}{\partial t}=\frac{1}{r} \frac{\partial}{\partial r}\left(r \frac{\partial g_{1}}{\partial r}\right)-\beta g_{1}-\left(\mathbf{P e} u+K_{1}\right), \\
\frac{\partial g_{2}}{\partial t}=\frac{1}{r} \frac{\partial}{\partial r}\left(r \frac{\partial g_{2}}{\partial r}\right)-\beta g_{2}-\left(\mathbf{P e} u+K_{1}\right) g_{1}+\left(1-K_{2}\right) \\
\frac{\partial g_{k+2}}{\partial t}=\frac{1}{r} \frac{\partial}{\partial r}\left(r \frac{\partial g_{k+2}}{\partial r}\right)-\beta g_{k+2}-\left(\mathbf{P e} u+K_{1}\right) g_{k+1}+\left(1-K_{2}\right) f_{k} \\
-\sum_{i=3}^{k+2} K_{i} g_{k+i-2} \quad(k=1,2, \mathrm{~L})
\end{gathered}
$$

The initial and boundary conditions on $g_{k}^{\prime s}$ are prescribed as follows:

$$
\begin{gathered}
g_{i}(0, r)=\delta_{0 i}, \quad(i=0,1,2) \\
\frac{\partial g_{i}}{\partial r}=0 \quad(i=0,1,2) \quad \text { at } \quad r=1, \\
\frac{\partial g_{i}}{\partial r}=0, \quad(i=0,1,2) \quad \text { at } \quad r=0,
\end{gathered}
$$

It may be inferred that:

$$
\int_{0}^{1} r \cdot g_{i}(r, t) d r=0
$$

The coefficients $K_{1}$ and $K_{2}$ can be derived by taking the cross-sectional averages as:

$$
\begin{gathered}
K_{1}(t)=-\operatorname{Pe} \bar{u}, \\
K_{2}(t)=1-2 \operatorname{Pe} \int_{0}^{1} r g_{1} u d r .
\end{gathered}
$$




$$
K_{i+2}(t)=-2 \operatorname{Pe} \int_{0}^{1} r g_{i+1} u d r
$$

$\bar{u}$ in Eqn. (44) is average velocity given by:

$$
\begin{aligned}
\bar{u} & =2 \int_{0}^{\eta} r v_{1} d r+2 \int_{\eta}^{1} r v_{2} d r \\
& =\left(\frac{1-N}{8-4 N}-\frac{\lambda}{8}\right) \eta^{4}-\frac{N(1-N) \eta^{2}}{s^{2}(2-N)}+\frac{\lambda}{8}+\frac{2 \eta N(1-N)}{s^{3}(2-N)} \frac{I_{1}(s \eta)}{I_{0}(s \eta)}
\end{aligned}
$$

\subsection{Dispersion coefficient:}

In order to solve Eq. (37) with the given initial and boundary conditions (Eqs. (40) -(42), one may deploy finite Hankel transforms defined by:

$$
\tilde{g}_{1}\left(t ; p_{i}\right)=\mathscr{H}\left\{g_{1}\right\}=\int_{0}^{1} r g_{1} J_{0}\left(p_{i} r\right) d r .
$$

Where $J_{0}$ is the Bessel function of order zero and $p_{i}$ is the positive roots of $J_{1}\left(p_{i}\right)=0$.

The corresponding inverse transform:

$$
g_{1}(t, r)=\mathscr{H}^{-1}\left\{\tilde{g}_{1}\left(t ; p_{i}\right)\right\}=\sum_{i=0}^{\infty} \frac{2 J_{0}\left(p_{i} r\right)}{J_{0}^{2}\left(p_{i}\right)} \tilde{g}_{1}\left(t ; p_{i}\right)
$$

Taking the Hankel transform of Eq. (37) and Eq. (40) with the aid of Eqs. (41) and (42) gives:

$$
\begin{gathered}
\frac{\partial \tilde{g}_{1}}{\partial t}+\left(\beta+p_{i}^{2}\right) \tilde{g}_{1}=-I_{1 n} \\
\tilde{g}_{1}\left(0 ; p_{i}\right)=0
\end{gathered}
$$

Here:

$$
I_{1 n}=\operatorname{Pe} \int_{0}^{1} r(u-\bar{u}) J_{0}\left(p_{i} r\right) d r
$$

Solving Eq. (50) subject to the initial condition (51), we obtain:

$$
\tilde{g}_{1}\left(t ; p_{i}\right)=\frac{\exp \left(-\left(\beta+p_{i}^{2}\right) t\right)-1}{\beta+p_{i}^{2}} I_{1 n}
$$


Hence,

$$
g_{1}(t, r)=\sum_{i=0}^{\infty} \frac{2 J_{0}\left(p_{i} r\right)}{J_{0}^{2}\left(p_{i}\right)} \frac{\exp \left(-\left(\beta+p_{i}^{2}\right) t\right)-1}{\beta+p_{i}^{2}} I_{1 n}
$$

Using Eq. (45) and (53), we obtained the dispersion coefficient with reaction and without reaction $(\beta=0)$ as:

$$
K_{2}=1-\sum_{i=0}^{\infty} \frac{\exp \left(-\left(\beta+p_{i}^{2}\right) t\right)-1}{\beta+p_{i}^{2}} I_{1 n} I_{2 n} .
$$

Where,

$$
I_{2 n}=4 \mathrm{Pe} \int_{0}^{1} r u \frac{J_{0}\left(p_{i} r\right)}{J_{0}^{2}\left(p_{i}\right)} d r
$$

Additionally, due to bulk degradation, the rate of decrease is further assessed

\subsection{Mean and Transverse Concentration}

The expression for the higher-order term in the series solutions are negligible (see- Wang and Chen [50]) and at the large time $t \geq 3$ only $g_{1}(r, t)$ is sufficient to estimate the solute concentration. Therefore, by neglecting the $K_{3}$ onwards we get:

$$
\frac{\partial \bar{C}}{\partial t}=-\operatorname{Pe} \bar{u} \frac{\partial \bar{C}}{\partial z}+K_{2}(t) \frac{\partial^{2} \bar{C}}{\partial z^{2}} .
$$

The solution of mean concentration w.r.t. the initial boundary condition given earlier is:

$$
\bar{C}=\frac{1}{2 \sqrt{\pi \xi(t)}} \exp \left(-\frac{(z-\mathrm{Pe} \bar{u} t)^{2}}{4 \xi(t)}\right)
$$

where,

$$
\xi(t)=\int_{0}^{t} K_{2}(s) d s
$$

The transverse concentration is obtained as: 


$$
C(\tau, r, z)=\bar{C}+\sum_{i=0}^{\infty} g_{i}(r, t) \frac{\partial^{i} \bar{C}}{\partial z^{i}}
$$

However, with the progress of time, the higher-order terms of the series become negligible. Wang and Chen [46] have shown that after time $t \geq 3$, only the first term of the series is sufficient i.e.:

$$
C(\tau, r, z)=\bar{C}+g_{1}(r, t) \frac{\partial \bar{C}}{\partial z}
$$

In the plots described in the next section, we use a specific definition for the rate of decrease of dispersion coefficient due to coupling number $(N)$ i.e.

\section{RESULTS AND DISCUSSION}

Figures 2- 9 illustrate the impact of micropolar coupling number $(N)$, micropolar parameter $(s)$, viscosity ratio (ratio of micropolar viscosity to Newtonian viscosity) (i.e.

$\lambda$ ) and reaction rate parameter $\beta$ on linear (axial) velocity ( $u$ i.e. $v_{1}, v_{2}$ in the core and plasma regions), time variation of dispersion coefficient $\left(K_{2}\right)$, axial and transverse mean concentration distributions. Generally, we prescribe high Péclet number $(P e=100$ i.e. convection dominates diffusion) and radius ratio is set as $\eta=0.95$.
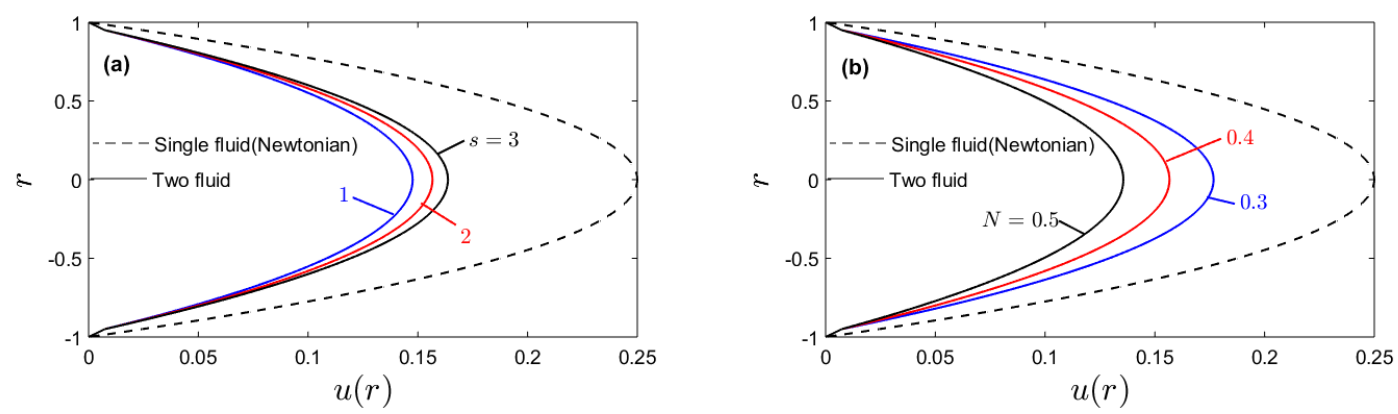


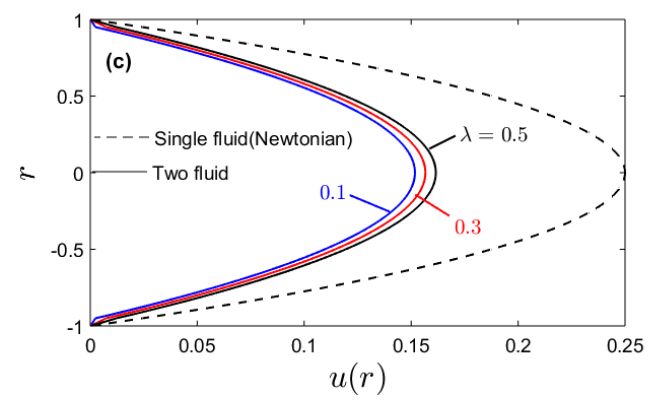

Fig 2. Dimensionless velocity profile for a variety of (a) micropolar coupling number $(N)$ where $(s=2, \lambda=0.3)$ ; (b) micropolar material parameter $(s)$ where $(N=0.4, \lambda=0.3)$; (c) viscosity ratio $(\lambda)$ where $(s=2, N=0.4)$..

Figures 2(a-c) illustrate the linear velocity variation with a radial coordinate for several parameters. In all cases, a substantially greater maximum velocity at the vessel centreline $(r=0)$ is computed with Newtonian single fluid compared with the two-fluid micropolarNewtonian scenario. An increase in micropolar parameter $(s)$, as observed in Fig. 2a, strongly boosts the linear velocity, i.e., induced significant flow acceleration. Conversely, increasing micropolar coupling number $(N)$, as visualized in Fig. $2 \mathrm{~b}$ damps the velocity i.e., produces substantial deceleration across the tube cross-section. Increasing viscosity ratio (ratio of micropolar viscosity to Newtonian viscosity) (i.e., $\lambda$ ) however, results in a marked acceleration in the flow. Clearly, greater micropolar (core region) viscosity relative to Newtonian viscosity enhances velocity magnitudes. In all cases smooth parabolic topologies are computed across the vessel cross-section, and there is no backflow, i.e., flow reversal does not arise.
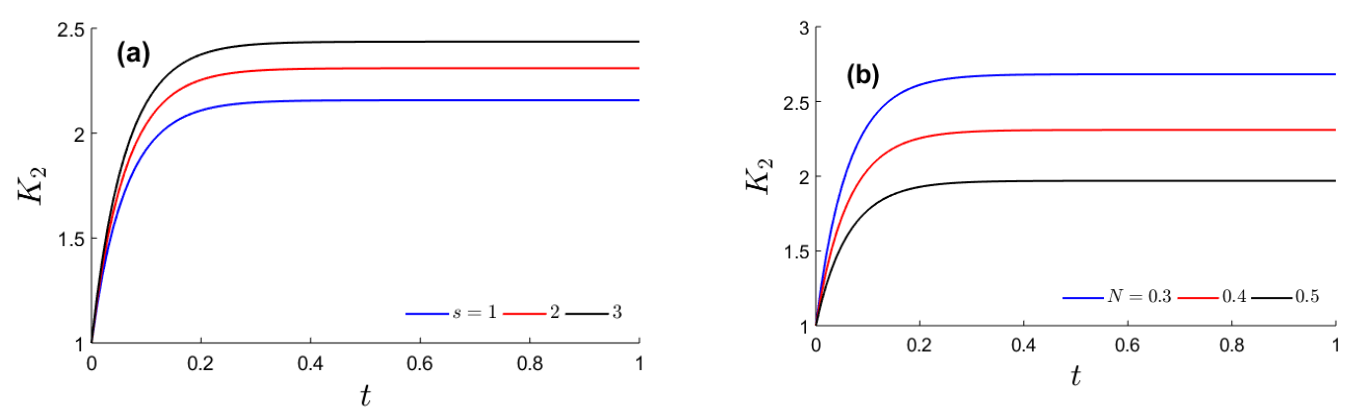

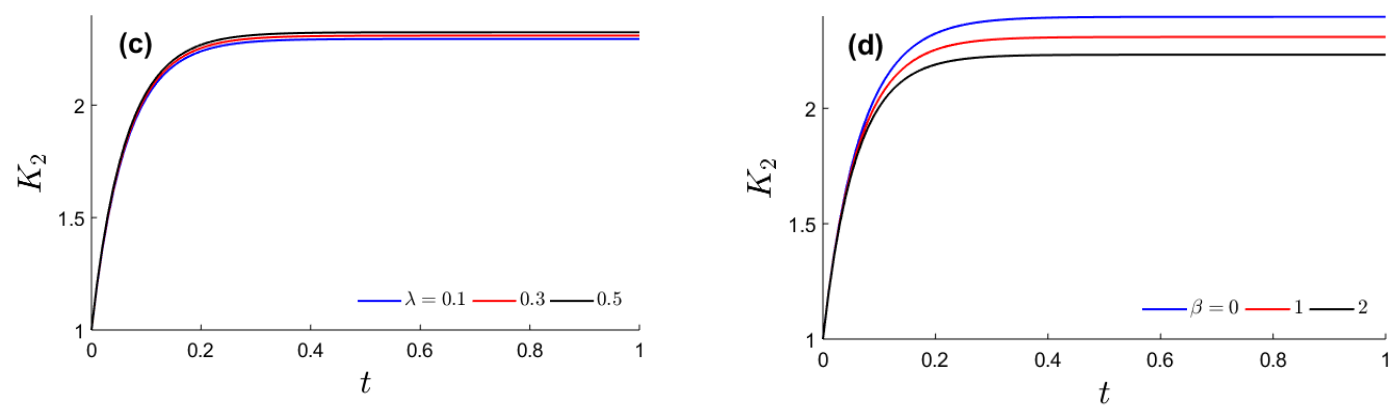

Fig 3. Time variation of dispersion coefficient $\left(K_{2}\right)$ for a variety of (a) micropolar coupling number $(N)$ where $(s=2, \lambda=0.3, \beta=1)$; (b) micropolar material parameter $(s)$ where $(N=0.4, \lambda=0.3, \beta=1)$; (c) viscosity ratio $(\lambda)$ where $(s=2, N=0.4, \beta=1)$; (d) reaction parameter $(\beta)$ where $(N=0.4, \lambda=0.3, s=2)$..

Figures 3a-d depict the transient variation in dispersion coefficient $\left(K_{2}\right)$ with all key parameters. In all plots, the dispersion coefficient ascends very steeply from initiation and eventually attains a plateau at large time (steady state). With increasing micropolar material parameter $(s)$, there is a distinct elevation in the dispersion coefficient (Fig. 3a) at all times $(t)$. However, the dispersion coefficient is suppressed with increasing micropolar coupling number $(N)$ as observed in Fig. 3b. A weak enhancement in the dispersion coefficient accompanies an increase in the viscosity ratio (ratio of micropolar viscosity to Newtonian viscosity) (i.e., $\lambda$ ) as seen in Fig. 3c. However, a strong depletion in the dispersion coefficient is induced with increasing bulk reaction parameter $(\beta)$, although the modification is less prominent than with increasing micropolar coupling number $(N)$.
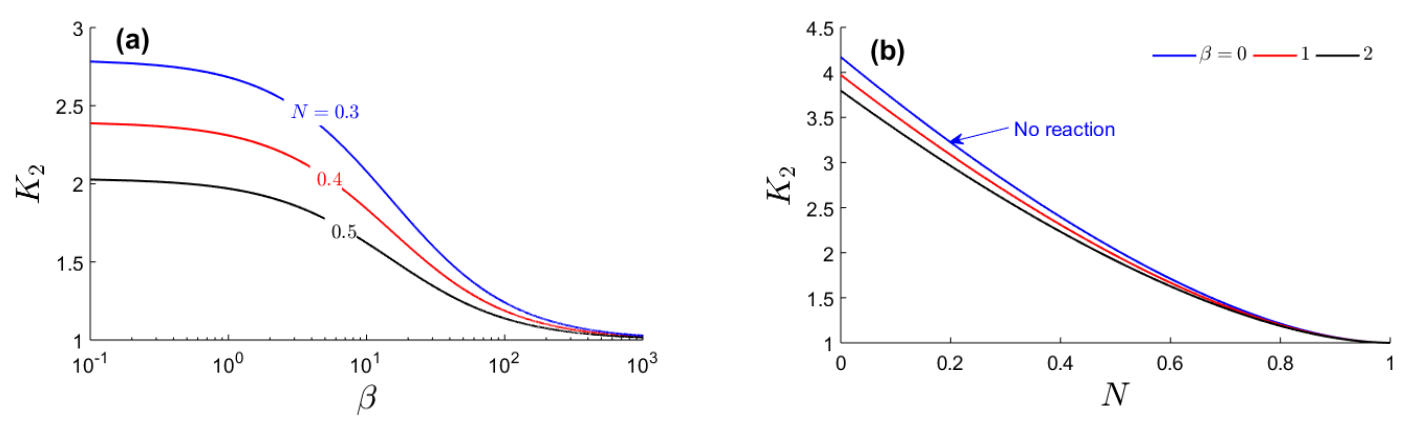

Fig 4. Plots of dispersion coefficient $\left(K_{2}\right)$ at the asymptotic time $(t=1)$ with (a) reaction rate parameter $(\beta) ;(\mathrm{b})$ micropolar coupling number $(N)$, where, $s=2$, and $\lambda=0.3$. 
Figures 4a-d depict the distribution of dispersion coefficient $\left(K_{2}\right)$ with several key parameters, computed at the asymptotic time $t=1$. In Fig. 4a it is evident that a smooth decay is produced in dispersion coefficient magnitudes with an increase in bulk reaction parameter $(\beta)$ and at very high values of this parameter, all profiles converge to the asymptotic limiting value of unity. Increasing the micropolar coupling parameter $(N)$ also results in a strong depletion in dispersion coefficient values. The maximum dispersion coefficient is computed in the absence of chemical bulk reaction $\beta=0$. Figure $4 \mathrm{~b}$ confirms this trend in which it is observed that the maximum dispersion coefficient is computed for the "no reaction" scenario. Quite a notable reduction in dispersion coefficient is clearly associated with increasing bulk reaction parameter $(\beta)$ consistent with [53] and also increment in micropolar coupling parameter $(N)$. In the limit of large $N$, the dispersion coefficient attains unity value.
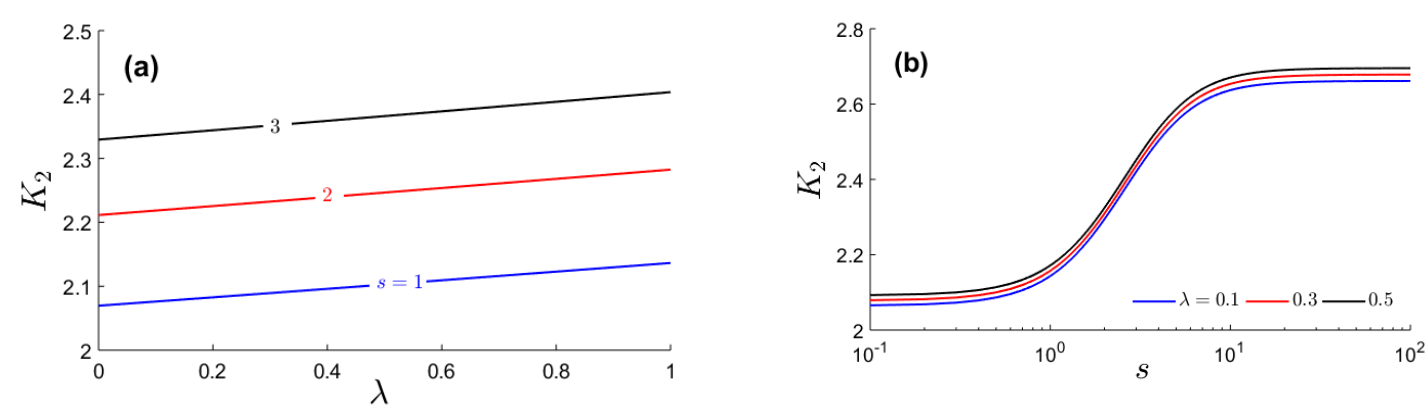

Fig 5. Plots of dispersion coefficient $\left(K_{2}\right)$ at the asymptotic time $(t=1)$ with (a) viscosity ratio $(\lambda)$; (b) micropolar material parameter $(s)$, where, $N=0.4$, and $\beta=1$.

Figures 5a-b illustrate the evolution in dispersion coefficient $K_{2}$ with (a) viscosity ratio

(ג) (b) micropolar parameter $(s)$ again at asymptotic time $(t=1)$. In both plots the bulk reaction parameter us constrained as unity $(\beta=1)$. A linear growth in dispersion coefficient $\left(K_{2}\right)$ accompanies increasing values of viscosity ratio, as plotted in Fig. 5a. However, there is a strong decrement in dispersion coefficient with increasing micropolar 
material parameter $(s)$. Clearly the micropolar core flow characteristics have a substantial impact on hydrodynamic dispersion of the solute in the regime. Fig. $5 \mathrm{~b}$ shows that a sigmoidal variation in dispersion coefficient is computed with increasing micropolar parameter.
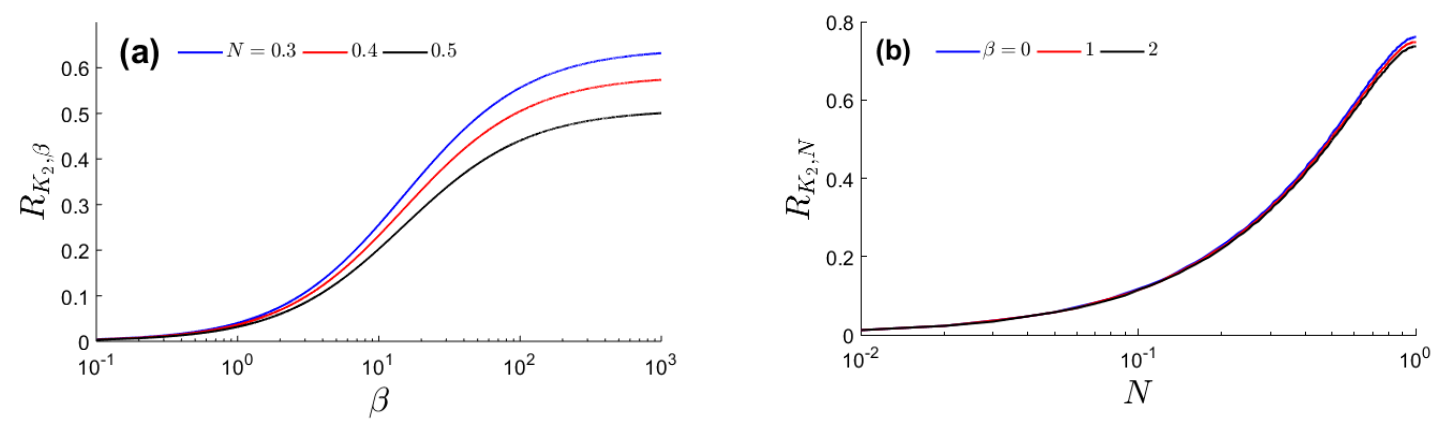

Fig 6. Variation of (a) $R_{K_{2}, \beta}$ with $\beta$, and (b) $R_{K_{2}, N}$ with $N$, at the asymptotic time $t=1$, where $s=2$, and $\lambda=0.3$.

The increment and decrement of dispersion coefficient as we seen Figs. 5 and 6 due to $\beta, N$ and $\lambda$ is further assessed by defining $R_{K_{2}, \beta}$ as:

$$
R_{K_{2}, \beta}=1-\frac{K_{2}}{\left.K_{2}\right|_{\beta=0}}
$$

$R_{K_{2}, N}$ as:

$$
R_{K_{2}, N}=1-\frac{K_{2}}{\left.K_{2}\right|_{N=0}}
$$

And $R_{K_{2}, \lambda}$ as:

$$
R_{K_{2}, \lambda}=1-\frac{K_{2}}{\left.K_{2}\right|_{\lambda=0}}
$$

The parameter $R_{K_{2}, \beta}$ and $R_{K_{2}, N}$ determine rate of decrement of dispersion coefficient due to reaction rate constant and micropolar coupling number respectively, whereas, $R_{K_{2}, \lambda}$ estimate the rate of increment of dispersion coefficient due to viscosity ratio. These 
incremental and decremented rate of dispersion coefficients w.r.t. the associated parameter are displayed through Figs. 6a-b and 7.

A strong increase in $R_{K_{2}, \beta}$ is induced with higher values of reaction parameter, $\beta$, as computed in Fig. 6a. The dispersion coefficient rate therefore grows with more intense first order chemical reaction in the blood flow. $R_{K_{2}, \beta}$ is however noticeably depressed with increasing micropolar coupling number, $N$ at all values of $\beta$. Fig. $6 \mathrm{~b}$ shows that $R_{K_{2}, N}$ however increases significantly with $N$; on the other hand, it is weakly reduced with increasing bulk reaction effect $(\beta)$ and this behaviour is most prevalent at high $N$ values.

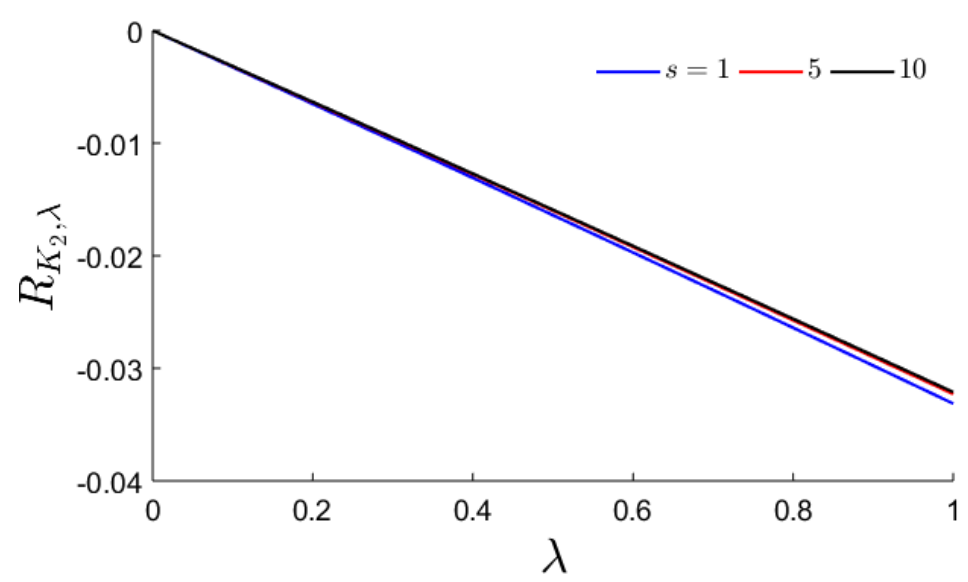

Fig 7. Variation of $R_{K_{2}, \lambda}$ with $\lambda$, and at the asymptotic time $t=1$, where, $N=0.4$, and $\beta=1$.

Figure 7 depicts a linear decay of $R_{K_{2}, \lambda}$ with increasing viscosity ratio. In other words the dispersion coefficient rate $R_{K_{2}, \lambda}$ is suppressed with increasing micropolar viscosity (core blood flow) relative to Newtonian viscosity (plasma peripheral layer). Conversely a non-trivial increment in dispersion coefficient rate $R_{K_{2}, \lambda}$ is induced with a rise in micropolar material parameter $(s)$. Maximum dispersion coefficient rate $R_{K_{2}, \lambda}$ is 
therefore achieved with minimal viscosity ratio and strong micropolarity of the core blood flow.
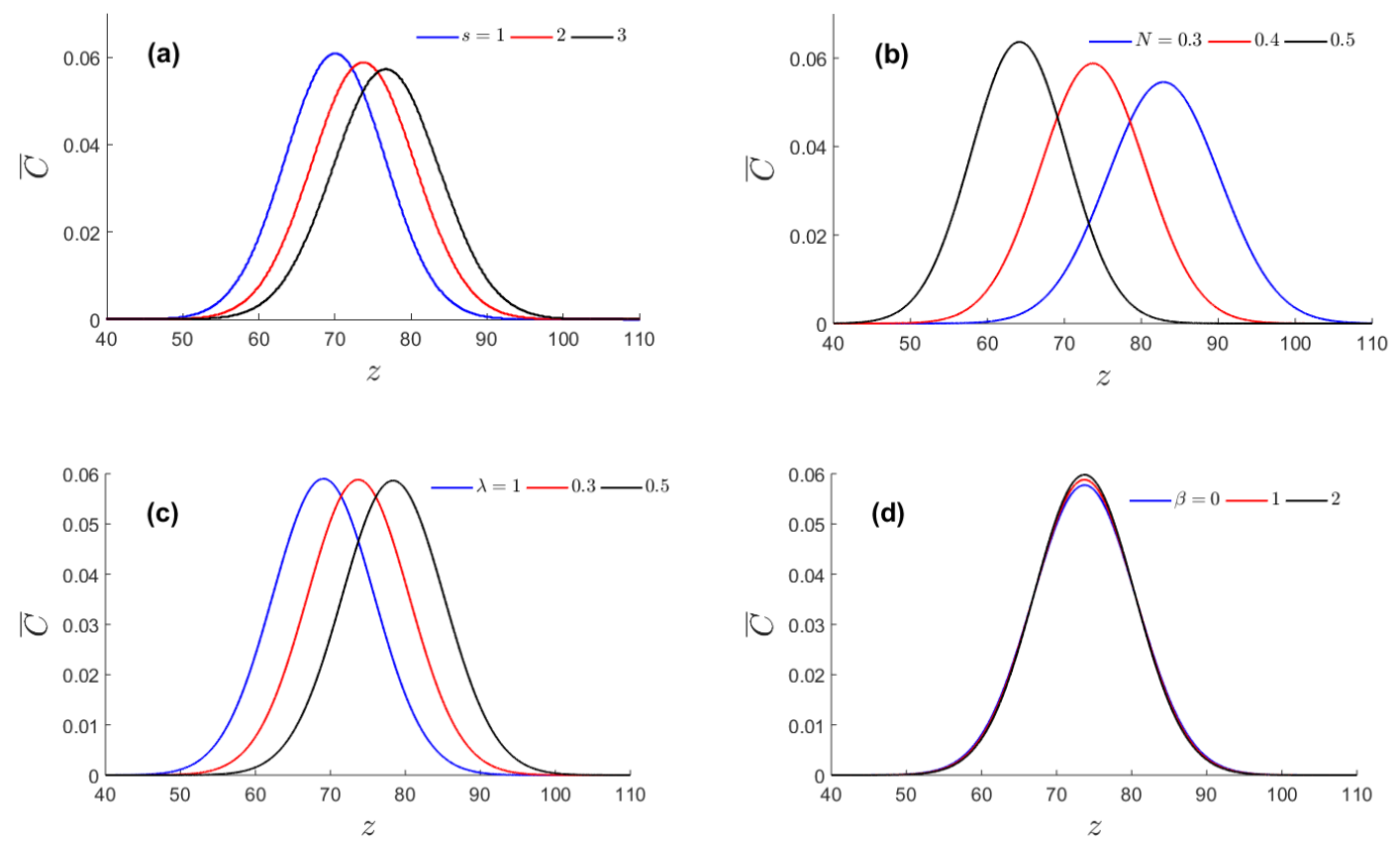

Fig 8. Axial mean concentration at the dimensionless time $\tau=10$ with $\mathrm{Pe}=100$, for a variety of (a) micropolar coupling number $(N)$ where $(s=2, \lambda=0.3, \beta=1)$; (b) micropolar material parameter $(s)$ where $(N=0.4, \lambda=0.3, \beta=1)$; (c) viscosity ratio $(\lambda)$ where $(s=2, N=0.4, \beta=1)$; (d) reaction parameter $(\beta)$ where $(N=0.4, \lambda=0.3, s=2)$..

Figures 8a-d visualize the evolution in axial mean concentration at the dimensionless time $t=10$ with axial coordinate $(z)$, for the convection-dominated case i.e. $\mathrm{Pe}=100$, for different values of the control parameters. Axial mean concentration peaks are decreased in magnitude (Fig. 8a) and the centre of gravity of the species migrate further along the arterial geometry with greater micropolar material parameter $(s)$ values. Symmetric parabolic (bell shaped) distributions are consistently computed. Figure $8 \mathrm{~b}$ shows that the opposite effect is induced with greater micropolar coupling number $(N)$ i.e. the peaks are elevated in magnitude but displaced backwards along the axial direction, although parabolic topologies are again observed. A slight increase in axial mean concentration peak value is caused with increasing reaction parameter. Figure $8 \mathrm{c}$ indicates 
that, although no tangible modification in axial concentration peak values is caused with an increase in viscosity ratio $(\lambda)$, the peaks are however translocated further along the axial direction. With increasing reaction parameter $(\beta)$, Fig. 8d displays that whereas there is no displacement of peaks along the axial direction, there is a slight enhancement in axial concentration peak magnitudes i.e., the minimum peak axial concentration corresponds to the "no reaction" case $(\beta=0)$.
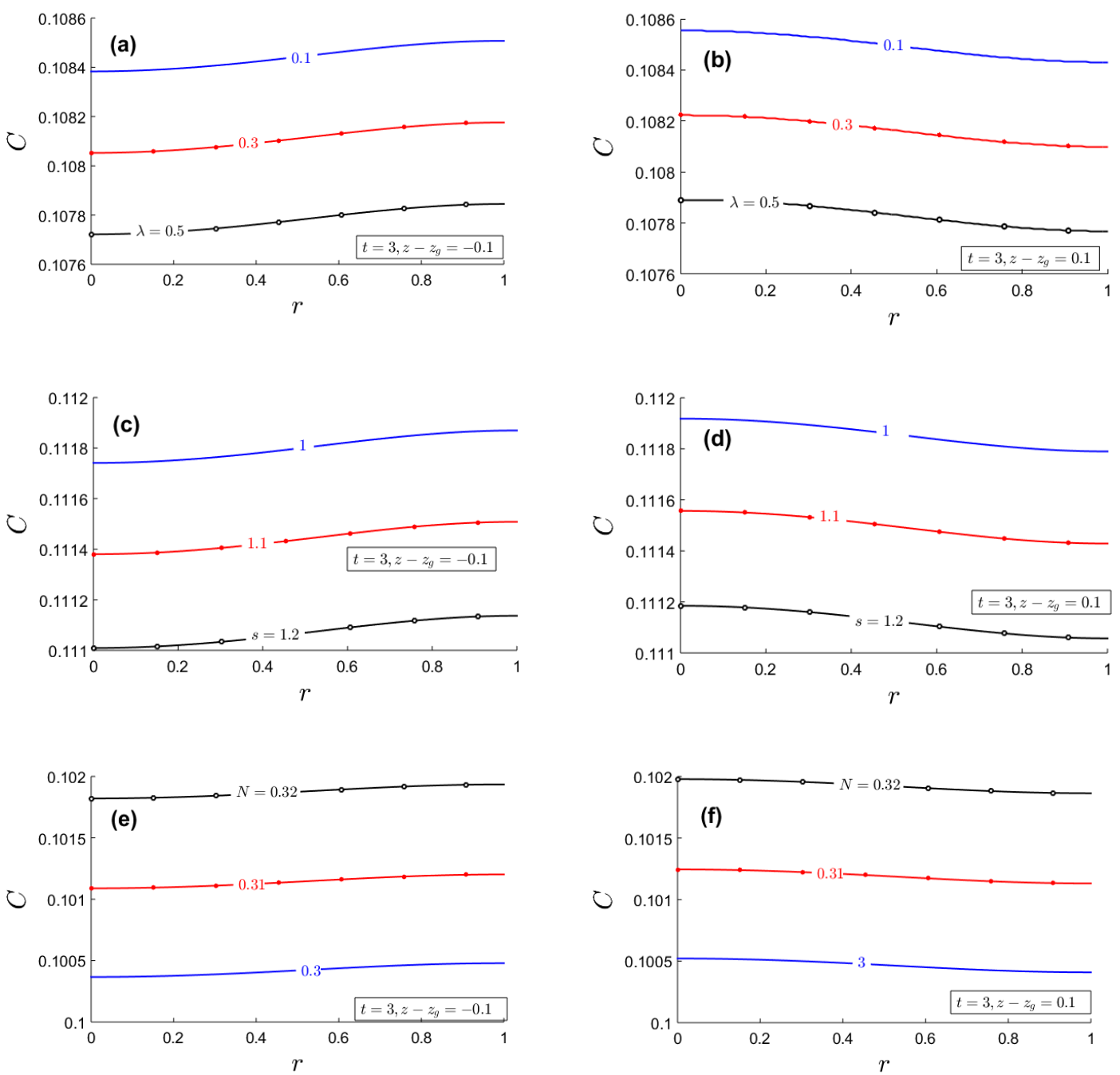

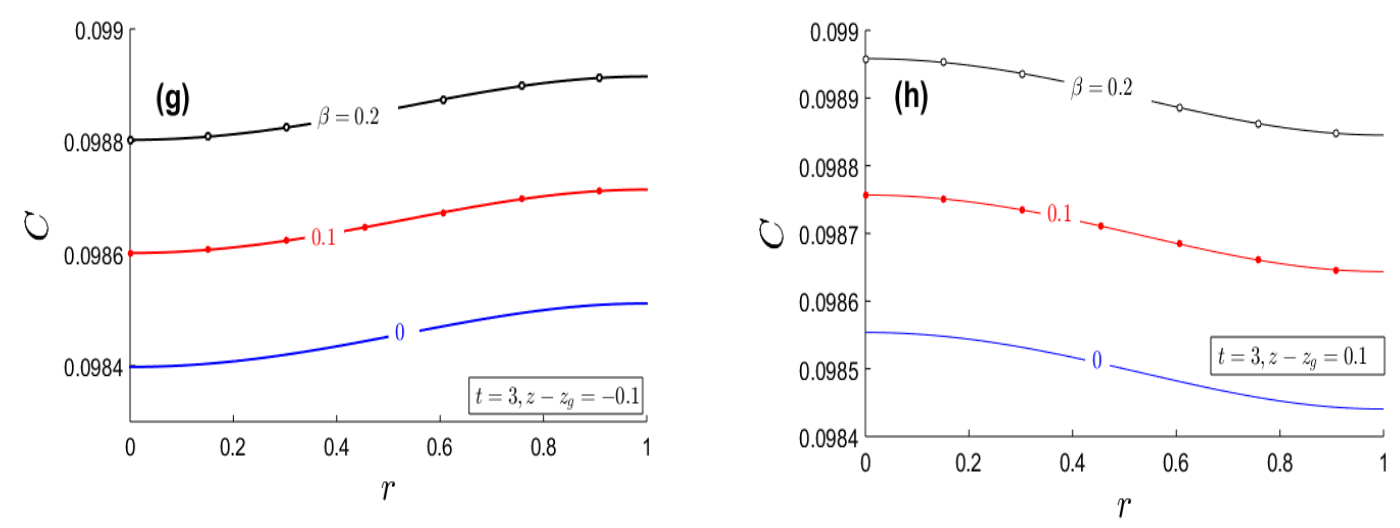

Fig 8. Transverse concentration at the different axial locations at the time $t=3$ for various (a, b) viscosity ratio $(\lambda)$ where $(s=2, N=0.4, \beta=1)$; (c, d) micropolar material parameter $(s)$ where $(N=0.4, \lambda=0.3, \beta=1) ;(\mathbf{e}, \mathbf{f})$ micropolar coupling number $(N)$ where $(s=2, \lambda=0.3, \beta=1)$; (g, h) reaction parameter $(\beta)$ where $(N=0.4, \lambda=0.3, s=2) \cdot$.

Finally, Figs 9a-h visualize the transverse concentration distributions with radial coordinate for all key parameters. Plots are grouped in pairs for upstream $\left(z-z_{g}=-0.1\right)$ and downstream $\left(z-z_{g}=0.1\right)$. Here, $z_{g}=\operatorname{Pe} \bar{u} t$ signifies the center of gravity of the reactive species which is moving at the average speed of the blood in the downstream direction in the blood vessel. Figures 9a, b show that transverse concentration increases with radial coordinate in upstream (column 1) whereas it decreases with radial coordinate in downstream (column 2). In both plots there is a strong depletion in transverse concentration of the reactive species (e. g. oxygen) with greater viscosity ratio $(\lambda)$. With increasing micropolar material parameter $(s)$, as displayed in Figs. 9 c,d there is significant suppression in transverse concentration magnitudes. Figures 9 e, f indicate that with increasing micropolar coupling number $(N)$ there is a marked enhancement in transverse concentration at all values of radial coordinate i.e. anywhere across the blood vessel cross section. A very distinct elevation in transverse concentration is computed with increasing reaction parameter $(\beta)$ in Figs. $9 \mathrm{~g}$-h and this is consistent at all values of radial coordinate. 


\section{SUMMARY AND CONCLUSIONS}

A comprehensive mathematical model has been developed for axisymmetric, incompressible and fully developed hemodynamic transport (blood flow) in a rigid impermeable artery under constant axial pressure gradient with unsteady reactive hydrodynamic dispersion. A two-fluid model has been employed wherein the core region is simulated as an Eringen micropolar fluid and the plasma layer is analyzed as Newtonian viscous fluid. The diffusing species e.g. oxygen is assumed to experience a first-order chemical reaction with streaming blood. Analytical solutions are developed for the nondimensional boundary value problem. Closed-form solutions are presented for the velocity and micro-rotation (angular velocity of micro-elements) profiles and a Gill decomposition method is deployed for the concentration field. Expressions are derived for the dispersion coefficient with reaction and without reaction and also the mean and transverse concentration functions. Extensive graphical visualization of the impact of key rheological and reaction parameters on transport characteristics has been presented. The key findings of the present computations are:

(i) Transverse concentration is elevated with increasing micropolar coupling number and reaction rate whereas it is decreased with greater micropolar material parameter and viscosity ratio.

(ii) Axial mean concentration peaks are reduced in magnitude and displaced further along the arterial geometry with greater micropolar material parameter values whereas the opposite trend is observed with greater micropolar coupling number i.e., peaks are displaced backwards and enhanced in magnitude. 
(iii) A slight elevation in axial mean concentration peak value is produced with increasing reaction parameter although there is no displacement of peaks along the axial direction.

(iv) Dispersion coefficient is reduced with increasing micropolar coupling number whereas it is enhanced with greater viscosity ratio.

(v) Peak axial velocity is also enhanced with greater viscosity ratio although the two-fluid case always achieves much lower magnitudes than the single fluid (Newtonian) case.

(vi) Transverse concentration always increases with radial coordinate for the upstream radius ratio case whereas it decreases with radial coordinate for the downstream case.

(vii) Minimal peak axial concentration is computed for the "no reaction" case.

(viii) Dispersion coefficient linearly increases with $\lambda$; however, rate of increment $R_{K_{2}, \lambda}$ exhibits a linear decrease with increasing viscosity ratio.

(ix) A slight increment in dispersion coefficient rate $R_{K_{2}, \lambda}$ due to viscosity ratio is induced with elevation in micropolar material parameter. Maximum dispersion coefficient rate $R_{K_{2}, \lambda}$ due to viscosity ratio is therefore computed with minimal viscosity ratio and strong micropolarity of the core blood flow. The present analytical study has revealed some interesting dispersion and hemodynamic characteristics of relevance to coagulation and biochemical reactions in streaming blood. However, the blood vessel has been assumed to be rigid. Furthermore, only homogenous reactions have been addressed whereas much more complex multi-stage chemical reactions in blood may be investigated. Future investigations may also consider 
deformability of the blood vessel wall and furthermore may explore nanoparticle doping [54] which is of relevance to pharmacodynamic treatments of diseased arteries.

Declaration of competing interest: The authors declared no potential conflict of interest. Acknowledgement: The authors gratefully record their thanks to Mrs. Khush Bég for painstakingly editing the paper to such a presentable form.

\section{REFERENCES}

[1] H.L. Goldsmith, R. Skalak, Hemodynamics, Ann. Rev. Fluid Mech. 7 (1975) 213247.

[2] Y.I. Cho, M.P. Mooney, D.J. Cho, Hemorheological disorders in diabetes mellitus, J. Diabetes Sci. Technol. 2 (2008) 1130-1138.

[3] V.T. Turitto, Blood viscosity, mass transport, and thrombogenesis, Prog. Hemost. Thromb. 6 (1982) 139-177.

[4] A. Toth, J. Papp, M. Rabai, P. Kenyeres, Z. Marton, G. Kesmarky, I. Juricskay, H.J. Meiselman, K. Toth, The role of hemorheological factors in cardiovascular medicine, Clin. Hemorheol. Microcirc. 56 (2014) 197-204.

[5] C. Le Devehat, T. Khodabandehlou, M. Vimeux, Impaired hemorheological properties in diabetic patients with lower limb arterial ischaemia, Clin. Hemorheol. Microcirc. 25 (2001) 43-48.

[6] K. Mori, H. Arai, K. Nakajima, A. Tajima, M. Maeda, Hemorheological and hemodynamic analysis of hypervolemic hemodilution therapy for cerebral vasospasm after aneurysmal subarachnoid hemorrhage, Stroke. 26 (1995) 16201626.

[7] C.-Y. Chang, H.-J. Liang, S.-Y. Chow, S.-M. Chen, D.-Z. Liu, Hemorheological Mechanisms in Alzheimer's Disease, Microcirculation. 14 (2007) 627-634.

[8] H.H. Yeh, Biomechanical modeling and hemorheological assessment of ascending thoracic aortic aneurysm, aortic heart valve, and blood clot, University of British Columbia, 2019. 
[9] J.-E. Yi, H.-J. Youn, Hemorheological abnormalities and their associations with coronary blood flow in patients with cardiac syndrome $\mathrm{X}$ : a comparison between males and females, Perfusion. 32 (2017) 57-67.

[10] O.K. Baskurt, H.J. Meiselman, Blood Rheology and Hemodynamics, Semin. Thromb. Hemost. 29 (2003) 435-450.

[11] J.F. Stoltz, M. Singh, P. Riha, Hemorheology in Practice, IOS Press, 1999.

[12] R. Skalak, N. Ozkaya, T.C. Skalak, Biofluid Mechanics, Annu. Rev. Fluid Mech. 21 (1989) 167-200.

[13] R.P. Chhabra, J.F. Richardson, Non-Newtonian Flow: Fundamentals and Engineering Applications, Elsevier, 1999.

[14] A.M. Robertson, A. Sequeira, R.G. Owens, Rheological models for blood, in: L. Formaggia, A. Quarteroni, A. Veneziani (Eds.), Cardiovasc. Math. Model. Simul. Circ. Syst., Springer Milan, Milano, 2009: pp. 211-241.

[15] O. Anwar Bég, S.K. Ghosh, S. Ahmed, T. Bég, Mathematical modeling of oscillatory magneto-convection of a couple-stress biofluid in an inclined rotating channel, J. Mech. Med. Biol. 12 (2012) 1250050.

[16] R.V. Sagayamary, R. Devanathan, Steady flow of couple stress fluid through tubes of slowly varying cross-sections - Application to blood flows, Biorheology. 26 (1989) 753-769.

[17] A.C. Eringen, Simple microfluids, Int. J. Eng. Sci. 2 (1964) 205-217.

[18] M.N.L. Narasimhan, A mathematical model of pulsatile flows of microstretch fluids in circular tubes, Int. J. Eng. Sci. 41 (2003) 231-247.

[19] D. Philip, P. Chandra, Flow of Eringen fluid (simple microfluid) through an artery with mild stenosis, Int. J. Eng. Sci. 34 (1996) 87-99.

[20] A.C. Eringen, Theory of Micropolar Fluids, J. Math. Mech. 16 (1966) 1-18.

[21] P. Chaturani, V.S. Upadhya, On micropolar fluid model for blood flow through narrow tubes, Biorheology. 16 (1979) 419-428.

[22] P. Chaturani, S.P. Mahajan, Poiseuille flow of micropolar fluid with non-zero couple stress at boundary with applications to blood flow, Biorheology. 19 (1982) 507-518.

[23] G. Akay, A. Kaye, Numerical solution of time dependent stratified two-phase flow of micropolar fluids and its application to flow of blood through fine capillaries, Int. J. Eng. Sci. 23 (1985) 265-276.

[24] P. Chaturani, V. Palanisamy, Microcontinuum model for pulsatile blood flow through a stenosed tube, Biorheology. 26 (1989) 835-846. 
[25] K.S. Mekheimer, Y.A. Elmaboud, The influence of a micropolar fluid on peristaltic transport in an annulus: application of the clot model, Appl. Bionics Biomech. 5 (2008) 13-23.

[26] O. Anwar Bég, R. Bhargava, S. Rawat, K. Halim, H.S. Takhar, Computational modeling of biomagnetic micropolar blood flow and heat transfer in a twodimensional non-Darcian porous medium, Meccanica. 43 (2008) 391-410.

[27] Sarifuddin, S. Chakravarty, P.K. Mandal, Heat transfer to micropolar fluid flowing through an irregular arterial constriction, Int. J. Heat Mass Transf. 56 (2013) 538551.

[28] M. Rashid, M. Keimanesh, O. Anwar Bég, T.K. Hung, Magnetohydrodynamic biorheological transport phenomena in a porous medium: A simulation of magnetic blood flow control and filtration, Int. J. Numer. Methods Biomed. Eng. 27 (2011) 805-821.

[29] Z. Asghar, N. Ali, O. Anwar Bég, T. Javed, Rheological effects of micropolar slime on the gliding motility of bacteria with slip boundary condition, Results Phys. 9 (2018) 682-691.

[30] M.K. Chaube, A. Yadav, D. Tripathi, O. Anwar Bég, Electroosmotic flow of biorheological micropolar fluids through microfluidic channels, Korea-Aust. Rheol. J. 30 (2018) 89-98.

[31] N.S. Akbar, D. Tripathi, Z.H. Khan, O. Anwar Bég, Mathematical modelling of pressure-driven micropolar biological flow due to metachronal wave propulsion of beating cilia, Math. Biosci. 301 (2018) 121-128.

[32] K. Rana, K.B. Neeves, Blood flow and mass transfer regulation of coagulation, Blood Rev. 30 (2016) 357-368.

[33] K.G. Mann, M.E. Nesheim, W.R. Church, P. Haley, S. Krishnaswamy, Surfacedependent reactions of the vitamin K-dependent enzyme complexes, Blood. 76 (1990) 1-16.

[34] K.G. Mann, K. Brummel-Ziedins, T. Orfeo, S. Butenas, Models of blood coagulation, Blood Cells. Mol. Dis. 36 (2006) 108-117.

[35] K.G. Mann, Biochemistry and physiology of blood coagulation, Thromb. Haemost. 82 (1999) 165-174.

[36] F. Shen, R.R. Pompano, C.J. Kastrup, R.F. Ismagilov, Confinement Regulates Complex Biochemical Networks: Initiation of Blood Clotting by "Diffusion Acting,” Biophys. J. 97 (2009) 2137-2145. 
[37] B. Zhao, K. Poonit, X. Zhou, C. Yao, C. Sun, H. Yan, The effect of chemical hemodynamic regulation on the survival of arterialized venous flaps, J. Plast. Surg. Hand Surg. 53 (2019) 83-88.

[38] C.-O. Ng, A note on the Aris dispersion in a tube with phase exchange and reaction, Int. J. Eng. Sci. 38 (2000) 1639-1649.

[39] S.N. Murthy, J. Feyen, Influence of variable permeability on the dispersion of a chemically reacting solute in porous media, Int. J. Eng. Sci. 27 (1989) 1661-1671.

[40] A.K. Roy, A.K. Saha, S. Debnath, Unsteady Convective Diffusion with Interphase Mass Transfer in Casson Liquid, Period. Polytech. Chem. Eng. 62 (2018) 215-223.

[41] A.K. Roy, A.K. Saha, S. Debnath, Hydrodynamic Dispersion of Solute under Homogeneous and Heterogeneous Reactions, Int. J. Heat Technol. 37 (2019) 387397.

[42] S. Debnath, A.K. Saha, B.S. Mazumder, A.K. Roy, Hydrodynamic dispersion of reactive solute in a Hagen-Poiseuille flow of a layered liquid, Chin. J. Chem. Eng. 25 (2017) 862-873.

[43] S. Debnath, A.K. Saha, B.S. Mazumder, A.K. Roy, Dispersion phenomena of reactive solute in a pulsatile flow of three-layer liquids, Phys. Fluids. 29 (2017)

[44] S. Debnath, A.K. Saha, P.G. Siddheshwar, A.K. Roy, On dispersion of a reactive solute in a pulsatile flow of a two-fluid model, J. Appl. Fluid Mech. 12 (2019) 9871000.

[45] P.G. Siddheshwar, S. Markande, Unsteady convective diffusion of solute in a micropolar fluid flow through a cylindrical tube, ZAMM - J. Appl. Math. Mech. Z. Für Angew. Math. Mech. 79 (1999) 821-833.

[46] V. Vidyanidhi, M.S. Murty, The dispersion of a chemically reacting solute in a micropolar fluid, Int. J. Eng. Sci. 14 (1976) 1127-1133.

[47] V.M. Soundalgekar, D.D. Haldavnekar, On the dispersion of a dye with a harmonically varying concentration in a channel flow of a micropolar fluid, Int. J. Eng. Sci. 27 (1989) 1527-1530.

[48] P.G. Siddheshwar, S. Manjunath, Unsteady convective diffusion with heterogeneous chemical reaction in a plane-Poiseuille flow of a micropolar fluid, Int. J. Eng. Sci. 38 (2000) 765-783.

[49] W.N.-N. Gill, G.I. Taylor, A note on the solution of transient dispersion problems, Proc. R. Soc. Lond. Ser. Math. Phys. Sci. 298 (1967) 335-339. 
[50] P. Wang, G.Q. Chen, Transverse concentration distribution in Taylor dispersion: Gill's method of series expansion supported by concentration moments, Int. J. Heat Mass Transf. 95 (2016) 131-141.

[51] G. Ravi Kiran, G. Radhakrishnamacharya, O. Anwar Bég, Peristaltic flow and hydrodynamic dispersion of a reactive micropolar fluid-simulation of chemical effects in the digestive process, J. Mech. Med. Biol. 17 (2016) 1750013.

[52] A.C. Eringen, Microcontinuum Field Theories II: Fluent Media, Appl. Mech. Rev. 55 (2002) B15-B15.

[53] A.K. Roy, A.K. Saha, S. Debnath, On Dispersion in Oscillatory Annular Flow Driven Jointly by Pressure Pulsation and Wall Oscillation, J. Appl. Fluid Mech. 10 (5) 1487-1500.

[54] Ankita Dubey, B. Vasu, O. Anwar Bég and Rama S R Gorla, Numerical simulation of two-fluid non-Newtonian nano-hemodynamics through a diseased artery with a stenosis and aneurysm, Computer Methods in Biomechanics and Biomedical Engineering (2019). DOI: 10.1080/10255842.2020.1729755 (28 pages) 